\title{
Invasive Egg Predators and Food Availability Interactively Affect Maternal Investment in Egg Chemical Defense
}

\section{OPEN ACCESS}

Edited by:

François Criscuolo,

Centre National de la Recherche Scientifique (CNRS), France

Reviewed by:

Ivan Gomez-Mestre,

Consejo Superior de Investigaciones

Cientificas (CSIC), Spain

Andreas Walzer

University of Natural Resources and

Life Sciences, Vienna, Austria

*Correspondence:

Sarah C. Pau

s.c.paul@exeter.ac.uk

${ }^{\dagger}$ Present Address Judith K. Pell,

J. K. Pell Consulting, Luton, United Kingdom

Specialty section:

This article was submitted to Behavioral and Evolutionary Ecology,

a section of the journal

Frontiers in Ecology and Evolution

Received: 09 October 2017

Accepted: 10 January 2018

Published: 30 January 2018

Citation:

Paul SC, Stevens M, Burton J, Pell JK, Birkett MA and Blount JD (2018) Invasive Egg Predators and Food Availability Interactively Affect Maternal Investment in Egg Chemical

Defense. Front. Ecol. Evol. 6:4.

doi: 10.3389/fevo.2018.00004

\author{
Sarah C. Paul ${ }^{1 *}$, Martin Stevens ${ }^{1}$, Jake Burton ${ }^{1}$, Judith K. Pell ${ }^{2 \dagger}$, Michael A. Birkett ${ }^{2}$ and \\ Jonathan D. Blount ${ }^{1}$ \\ ${ }^{1}$ College of Life and Environmental Sciences, Centre for Ecology and Conservation, College of Life and Environmental \\ Sciences, University of Exeter, Cornwall, United Kingdom, ${ }^{2}$ Rothamsted Research, West Common, Harpenden, \\ United Kingdom
}

Invasive species commonly predate the offspring of native species and eggs are the life stage most vulnerable to this predation. In many species with no maternal care, females can alter the phenotype of eggs to protect them, for instance through chemical defense. In ladybirds egg alkaloids deter predators, including invasive predatory species of ladybirds, but conversely may attract cannibals who benefit from the consumption of eggs with higher alkaloid levels. Invasive predators tend to be more abundant where resources are also abundant, but in high resource environments the maternal fitness benefits of sibling cannibalism are low. Consequently this presents a conflict for female ladybirds between the different factors that influence egg alkaloid level, as protecting her eggs from predators might come with the cost of inadvertently encouraging within-clutch cannibalism under circumstances where it is not beneficial. We investigated how the ladybird Adalia bipunctata addresses this trade-off experimentally, by measuring the quantity of alkaloids in eggs laid by ladybirds in environments that differed in levels of resource availability and perceived predation risk from an invasive predator Harmonia axyridis. Females did lay eggs with higher egg alkaloid levels under poor resource conditions, but only when predator cues were absent. The resulting negative correlation between egg number and egg alkaloid level under poor resource conditions indicates a trade-off between these two attributes of maternal investment, mediated by female response to offspring predation risk. This implies that selection pressures on mothers to adaptively adjust the risk of siblicide may outweigh the need to protect offspring from interspecific predation. Our results demonstrate that maternal effects are an important aspect of species' responses to invasive predators, and highlight the value of studying maternal effects in the context of the multifaceted environments in which they occur.

Keywords: maternal effects, chemical defense, invasive species, ladybirds, alkaloids

\section{INTRODUCTION}

Ecosystems across the globe are undergoing rapid anthropogenically driven change (Steffen et al., 2007), exposing species to novel biotic and abiotic pressures, for example invasive species (Mack et al., 2000). While there is a need to understand the threats that these changes pose, they also provide a unique opportunity to study how organisms adapt to novel alterations in 
their environment (Visser, 2008). Maternal effects enable the alteration of phenotypes across generations independent of genetic inheritance (Mousseau and Fox, 1998; Wolf and Wade, 2009), facilitating the rapid response of species to environmental change (Bernardo, 1996a). It is becoming increasingly apparent that such effects play a key role in the response of species to anthropogenic change e.g., increases in temperature (Donelson et al., 2012) and oceanic $\mathrm{CO}_{2}$ levels (Miller et al., 2012). In contrast, much less is known about how maternal effects may mediate species responses to novel invasive predators, a key component of global change (Mack et al., 2000).

Invasive species commonly prey upon the offspring of native species (e.g., Pell et al., 2008), and consequently can have profound effects on the abundance and persistence of native species (Paolucci et al., 2013). Their impact depends heavily upon how well they can detect and then respond appropriately to invasive predators (i.e., prey naïveté; Carthey and Banks, 2014). Naiveté is determined by the ecological novelty of the predator (Rehage et al., 2009), the diversity of native predators to which prey are exposed (Ferrari et al., 2007), and the adequacy of prey defense (Banks and Dickman, 2007). The expectation is that due to a lack of shared evolutionary history, native prey are likely to have high naïveté to invasive predators (Cox and Lima, 2006). However, where invasive predators produce similar visual and/or chemical cues to native predators, prey can adaptively adjust their behavior and morphology in response to invasive predator presence (Kovalenko et al., 2010). Mothers are already known to alter offspring phenotype, via maternal effects, in direct response to predation pressure and cues of native predator presence (Storm and Lima, 2010); with these changes in offspring phenotype maximizing maternal reproductive success (e.g., Walsh et al., 2015). It is conceivable therefore that, under certain conditions, maternal effects may also play a role in the response of native species to invasive predators.

Any transgenerational response to an invasive predator is unlikely to occur independently of maternal responses to other environmental factors. Females reproduce in complex multidimensional environments where positively covarying factors can have opposing influences on offspring survival and phenotype, e.g., predation risk and favorable abiotic conditions (Touchon and Worley, 2015). Consequently, focusing on maternal effects in the context of isolated single environmental variables shows only a small part of the picture (Lau et al., 2008). The effect of conflicting environmental factors on plastic phenotypes has been elegantly illustrated in studies of individual phenotypic plasticity, as opposed to the transgenerational phenotypic plasticity seen in maternal effects, in response to reliable environmental cues (Tollrian et al., 2015). Crossed gradients of environmental variables that favor opposing phenotypes typically result in individuals displaying a balance between the two phenotypic optima (Relyea, 2004; Hoverman and Relyea, 2016). It therefore seems reasonable to expect that changes in offspring phenotype, brought about by maternal effects, may themselves also be subject to antagonistic selection pressures on phenotypic optima. For example, increasing egg size may compensate for poor resource availability (Fox and Mousseau, 1996), but may also make offspring more conspicuous or attractive to parasites (Otto and Mackauer, 1998). Empirical tests of how such dynamic trade-offs influence maternal effects are however scarce, and even less is known about how they may be altered by anthropogenically driven environmental changes, e.g., the arrival of invasive species.

To fully understand female investment via maternal effects in the face of invasive offspring predators, reproductive decisions must therefore be studied in the context of the interactive and potentially antagonistic factors present in the mother's reproductive environment (Deas and Hunter, 2013, 2014). The 2-spot ladybird, Adalia bipunctata, is a native UK ladybird and an ideal species with which to investigate such ideas. Like other ladybird species, female $A$. bipunctata provision eggs with a species specific alkaloid defense chemical [(-)-adaline] that deters predators (Agarwala and Dixon, 1992), such as the larvae of the invasive ladybird Harmonia axyridis (Sato and Dixon, 2004). Conversely alkaloids, such as (-)-adaline, may also actually attract cannibals upon which there is no toxic effect and that benefit from the consumption of conspecific eggs with greater alkaloid concentration (Kajita et al., 2010). Invasive predators are more abundant where resources are also abundant (Smith and Gardiner, 2013), but in high resource environments, the maternal fitness benefits of sibling cannibalism are low (Pfennig, 1997). This presents a conflict for female ladybirds when responding to invasive offspring predators and to resource abundance, as protecting her eggs from predators might come with the cost of inadvertently encouraging within-clutch cannibalism under circumstances where it is not beneficial.

To assess the degree to which each of these antagonistic pressures determine female reproductive investment, we tested whether cues of invasive $H$. axyridis larvae and resource availability (aphid presence) interactively affect maternal investment in the ladybird A. bipunctata, including egg (-)adaline level. In a palatability experiment, we also assessed whether A. bipunctata eggs with a higher (-)-adaline content were more vulnerable to cannibalism by conspecific larvae. This is crucial because, while it has been demonstrated that ladybird eggs with high alkaloid content are less palatable to predators (Kajita et al., 2010), prior work, although highly indicative, does not explicitly test whether increasing alkaloid content increases egg palatability for cannibals. We predicted that: (1) In the risk-by-resource experiment (a) Females will be more likely to lay eggs in the presence of predators when aphids are also present; (b) Egg alkaloid level will be greatest when perceived predation risk, and the selective benefit of cannibalism, are at their highest (i.e., resources are low) and smallest under the reverse conditions. (2) In the cannibalism experiment, eggs with higher alkaloid content will be preferentially cannibalized.

\section{METHODS}

\section{Insect Cultures}

Stock cultures of A. bipunctata ladybirds, obtained from Gardening Naturally (Love Lane Industrial Estate, Cirencester, UK), and harlequin ladybirds, obtained from two well established wild UK invasive populations (collected at UK grid references SU6168 8950 and TL1253 1317) were maintained on an ad 
libitum diet of pea aphids (Acyrthosiphon pisum; reared on dwarf bean [Vicia faba] Sutton variety) at $18^{\circ} \mathrm{C}$ in a $16 \mathrm{~L}: 8 \mathrm{Dh}$ light:dark regime.

\section{Experimental Protocols Egg Laying Experiment}

Experimental individuals were 1 st generation virgin $A$. bipunctata adults of known age (19-29 days post eclosion) reared from stock culture individuals. Females were mated with a non-sib male (80 females and 80 males from five families) and $24 \mathrm{~h}$ after pairing, females were weighed to the nearest $0.1 \mathrm{mg}$ (analytical balance GR-200 A\&D ${ }^{\circledR}$ Gemini $^{\mathrm{TM}}$ ) before being placed individually into the experimental microcosm. As a mobile egg laying species with no parental care A. bipunctata can maximize reproductive success in the face of variation in factors that influence offspring survival via; laying site choice (Refsnider and Janzen, 2010) and the alteration of egg phenotype (non-genetic maternal effects; Marshall and Uller, 2007). The experimental microcosm was set up in order to enable the quantification of movement indicative of laying site choice as well as maternal investment, in response to variation in offspring predation risk and resource availability. The experimental microcosm $(38 \times 23 \times 17 \mathrm{~cm})$ contained two $V$. faba plants, the treatment plant and a second plant, both $12 \pm 2.5 \mathrm{~cm}$ in height and set $14 \mathrm{~cm}$ apart from each other and $7 \mathrm{~cm}$ from the tray edge. The treatment plants were manipulated so that they varied in aphid abundance and perceived predation risk, whereas the second plant was always clean, i.e., no aphids and no predator tracks $(\mathrm{A}-/ \mathrm{P}-)$.

There were four treatments on the treatment plant:

$(\mathrm{A}+/ \mathrm{P}-)$ aphids and no perceived predation risk,

$(\mathrm{A}+/ \mathrm{P}+)$ aphids and perceived predation risk,

$(\mathrm{A}-/ \mathrm{P}-)$ no aphids and no perceived predation risk,

$(\mathrm{A}-/ \mathrm{P}+)$ no aphids and perceived predation risk.

A+ plants were infested with 60 pea aphids of mixed instars 4 days prior to the experimental start date. In $\mathrm{P}+$ plants the perceived predation risk was achieved by attaching a filter paper (Fisherbrand QL100, $5 \mathrm{~cm}$ diameter) on which the tracks of harlequin larvae had been deposited (from two unfed larvae that had been allowed to walk on the filter paper in a dish for $12 \mathrm{~h}$; Carter and Dixon, 1982; Doumbia et al., 1998; Magro et al., 2007). These "larval tracks" are low volatility contact cues and as such have high temporal persistence Ruzicka, 2002; Oliver et al., 2006. Papers with tracks and control papers (clean filter papers for use on P-plants), were each cut into four strips. These were attached by wrapping the paper either around stems or by folding either side of and flush to a leaf and stapling the paper to itself, such that the plants remained undamaged.

Females from different sib clusters were distributed evenly between the four treatments, so that morph and family ID were represented equally. Final sample sizes were as follows, $\mathrm{A}+/ \mathrm{P}-$, $N=20 ; \mathrm{A}+/ \mathrm{P}+, N=18 ; \mathrm{A}-/ \mathrm{P}-, N=18 ; \mathrm{A}-/ \mathrm{P}+, N=20$, as four replicates failed due to escapees. Females were monitored for $9 \mathrm{~h}$ : every $15 \mathrm{~min}$ for the first $3 \mathrm{~h}$, every $30 \mathrm{~min}$ for the subsequent $3 \mathrm{~h}$, and every hour for the final $3 \mathrm{~h}$. Movement from the focal plant, the time of onset and location of egg laying, and the time that each of these behaviors was observed was recorded. Once they had laid, females and eggs were removed, and all eggs were frozen at $-80^{\circ} \mathrm{C}$ prior to toxin analysis. All observations were made in a controlled temperature room (Adcocks Cereal Growth Chamber 2007, Adcocks, UK) at $21^{\circ} \mathrm{C}$ in a $16 \mathrm{~L}: 8 \mathrm{Dh}$ light regime over 5 days, with four replicates of each treatment per day.

Adalia bipunctata eggs contain the toxic alkaloid (-)-adaline. To assay (-)-adaline, each egg was weighed to the nearest 1 $\mu \mathrm{g}$ using an electronic microbalance (Cahn C33; Scientific and Medical Products Ltd, Manchester, UK.) and homogenized for $30 \mathrm{~s}$ in $200 \mu \mathrm{l}$ of dichloromethane, using a handheld electronic pestle. Each sample was then centrifuged at 13RPM and $4^{\circ} \mathrm{C}$ for $10 \mathrm{~min} .100 \mu \mathrm{l}$ of solution was transferred into a screwtop auto-sampler vial. Samples $(2 \mu \mathrm{l})$ were analyzed on a nonpolar (HP-1, $50 \mathrm{~m} \times 0.32 \mathrm{~mm}$ inner diameter $\times 0.5)$ GasChromatograph (GC) (Agilent Technologies, UK) fitted with a cool-on-column injector, a deactivated HP-1 pre-column ( $1 \mathrm{~m} \times$ $0.53 \mathrm{~mm}$ inner diameter) and a flame ionization detector (FID). The GC oven temperature was maintained at $30^{\circ} \mathrm{C}$ for $1 \mathrm{~min}$ after sample injection and then raised by $5^{\circ} \mathrm{C} \min ^{-1}-150^{\circ} \mathrm{C}$, then $10^{\circ} \mathrm{C} \min ^{-1}-240^{\circ} \mathrm{C}$. The carrier gas was hydrogen. Peak enhancement by co-injection with a pure (-)-adaline standard (synthesized using the method of Hill and Renbaum, 1982) was used to confirm correct identification of the (-)-adaline peak. Absolute (-)-adaline concentration per egg ( $\mathrm{ng} / \mathrm{mg}$ ) was quantified by transforming the peak area using a calibration curve created from an external standard of pure (-)-adaline in dichloromethane at the following concentrations; 100, 50, 10, 5, and $1 \mathrm{ng} / \mu \mathrm{l}$.

\section{Cannibalism Experiment}

Recently eclosed 4th instar A. bipunctata larvae $(n=161)$ were raised from 14 pairs of adults taken from the stock culture and fed on an ad lib. diet of pea aphids. Larvae were fed $24 \mathrm{~h}$ prior to the trial with $(0.01 \mathrm{~g} / \sim 40$ aphids), to standardize hunger levels, weighed to the nearest $0.1 \mathrm{mg}$ (Ohaus Explorer ${ }^{\circledR}$ e12140 balance, Ohaus Europe GmbH, Greifensee, Switzerland) and then placed individually into test arenas $(55 \times 10 \mathrm{~mm}$ Petri dish) facing two eggs. The bidirectional choice test consisted of two conspecific eggs one with a high toxin content $(\mathrm{H})$ and the other a low toxin content (L), set $20 \mathrm{~mm}$ away from larvae. The $A$. bipunctata eggs used for the trial were collected from culture and frozen at $-80^{\circ} \mathrm{C}$ for 12 months. Quantification of egg ()-adaline content is destructive, but within-female repeatability of egg (-)-adaline content and egg mass is high (Paul et al., 2015). The values of egg (-)-adaline and egg mass from previously analyzed eggs (Paul et al. unpublished data) were therefore used as proxy values for the females that laid them and used to select remaining unanalysed eggs for the cannibalism trials. To maximize egg toxin difference between $\mathrm{H}$ and $\mathrm{L}$ eggs and minimize difference in egg mass, females were ranked based on these values and paired so that the values of (-)-adaline were more than four standard deviations (SD) apart and egg mass values were $<1 \mathrm{SD}$ apart. In the trial eggs were set $20 \mathrm{~mm}$ apart, the position of each egg was alternated between dishes to obviate side preference bias, and trials were carried out blindly with respect to the hypotheses under test and under 
uniform conditions (HQI lamps, $700 \mu$ mols $\mathrm{m}^{-2} \mathrm{~s}^{-1}$ at $18 \pm$ $2^{\circ} \mathrm{C}$ ). Larvae were continuously monitored and the time at which they contacted an egg, the identity of the egg first contacted, the identity of the egg consumed, whether they consumed the entire egg and the duration of feeding, were recorded. Larvae were then immediately euthanized and stored at $-80^{\circ} \mathrm{C}$. The body length of individuals was measured to the nearest $0.001 \mathrm{~mm}$ using a Leica M165C stereo microscope (Leica Micro Systems Ltd, Milton Keynes, UK), in order to enable the calculation of larvae body condition. This was calculated using the Scaled Mass Index following (Peig and Green, 2009, 2010), which fully accounts for the scaling relationship between mass and length.

\section{Data Analyses}

Data were analyzed using $\mathrm{R}$ version 3.2.2 (R Core Team, 2014). Data were examined for normality, homoscedasticity and outliers and where appropriate transformed to improve the normality of model residuals. The alpha level was set at 0.05 for all tests and stepwise backwards deletion was employed to reach the minimum adequate model (Crawley, 2014).

\section{Egg Laying Experiment}

All models contained perceived predation risk/absence of perceived risk $(\mathrm{P}+/ \mathrm{P}-)$, aphid presence/absence $(\mathrm{A}+/ \mathrm{A}-)$, and their interaction as fixed effects. Generalized linear models (GLM, package = MASS) with binomial errors and a log link function were used to assess whether these fixed effects and female age influenced: whether a female moved from the focal plant, whether females laid eggs, and whether or not they laid on the focal plant. The influence of the treatments and of female weight and age on the time of egg laying was assessed using a GLM with negative binomial errors and a log link function (error structure used to account for over-dispersion in each model). For those females that moved from the focal plant when aphids where absent and predator cues were present $(\mathrm{A}-/ \mathrm{P}+)$, this altered the laying environment of females in this treatment as "larval tracks" are low volatility contact cues (Ruzicka, 2002; Oliver et al., 2006). Analyses for reproductive investment [i.e., egg number and egg (-)-adaline level and concentration] were therefore carried out with predation risk level altered to $\mathrm{P}$ - if females in the $\mathrm{A}-/ \mathrm{P}+$ treatment moved from and laid eggs away from the focal plant. The influence of the treatments and of female weight and age on total egg number was assessed using a GLM with negative binomial errors and a log link function (error structure used to account for over-dispersion in each model). The effect of the treatments on the sqrt of both total egg alkaloid [(-)-adaline] level and egg alkaloid [(-)-adaline] concentration was fitted with the standard fixed effects mentioned above and GC run date, and female ID (multiple eggs per female were measured) as nested random effects using a general linear mixed effects model (LMER, package $=$ lme4; Bates et al., 2015). Egg mass was included as a factor in the aforementioned model for total egg alkaloid level to assess the relationship between egg size and alkaloid content. Post-hoc comparisons of significant interaction terms were carried out using the "multcomp" package in R (Hothorn et al., 2008). Additionally, due to results from the main analysis on egg (-)-adaline, a further analysis was carried out to assess the relationship between egg (-)-adaline and total egg number in the absence of aphids (i.e., $\mathrm{P}-/ \mathrm{A}-$ and $\mathrm{P}+/ \mathrm{A}$ - treatments) using a LMER where GC run date, and female ID were nested random effects. The repeatability of alkaloid levels in eggs laid by each female was calculated in the "rptR" package following (Nakagawa and Schielzeth, 2010).

\section{Cannibalism Experiment}

A two-tailed binomial test was used to assess whether there was a difference in the consumption of high toxicity $(\mathrm{H})$ and low toxicity (L) eggs in the bi-directional choice trials. A generalized linear mixed effects model (GLMM) with a binomial error structure, logit link, and larval family ID as a random effect was fitted to assess whether larval body condition, the egg first contacted, and time of day (fixed effects) predicted whether an $\mathrm{H}$ or $\mathrm{L}$ toxicity egg was consumed in the trial. The effect of egg toxicity and Scaled Mass Index on consumption latency was analyzed using GLMM with a Poisson error structure and log link function, where larval family ID and an observation level were included as random effects. The observational random level effect was included as time data were over dispersed but were not zero-inflated (Harrison, 2014).

\section{RESULTS}

\section{Egg Laying Experiment}

Contrary to predictions there was no interactive effect of the aphid and simulated predation risk (from now on predation risk) treatments on egg laying behavior (Table 1). A greater proportion of females laid eggs when aphids were present than when they were absent $(A+: 95 \%$ and $A-: 68 \%$, Table 1). Of females that laid eggs $(n=62)$, fewer laid on the focal plant when aphids were absent than when they were present $(A+: 97 \%$ and $A-$ : $62 \%$, Table 1). It also took females longer to initiate laying when aphids were absent than when they were present $(\mathrm{A}-$ : $349 \pm 35 \mathrm{~min}$ and $\mathrm{A}+: 223 \pm 23 \mathrm{~min}$, mean \pm SE; Table 1). There was a trend, though no significant, for females to lay more eggs when exposed to predation risk $(\mathrm{P}+)$ than when predation risk was absent $(\mathrm{P}-$ ) (Mean "Total number of eggs laid" \pm SE split by predation risk: $\mathrm{P}+=19.44 \pm 1.61, \mathrm{P}-$ $=14.80+1.21$; split by predation risk and aphid treatment: $\mathrm{P}+\mathrm{A}+=18.83 \pm 1.74, \mathrm{P}+\mathrm{A}-=21.00 \pm 3.81, \mathrm{P}-\mathrm{A}+=$ $15.00+1.43, \mathrm{P}-\mathrm{A}-=14.59 \pm 2.05)$, and under both treatments female fecundity increased with female mass (Figures 1, 2, Table 1).

There was high within-female repeatability for total egg (-)-adaline $[R=0.627,95 \% \mathrm{CI}=(0.451,0.737), p<0.001]$, and egg (-)-adaline concentration $[R=0.57,95 \% C I=(0.317$, $0.731), p<0.001]$, with confidence intervals well above zero. Both total egg (-)-adaline and egg (-)-adaline concentration were significantly higher when aphids were absent than when they were present but only when the risk of predation was absent [Figure 3, Table 1; P-A + and P-A - pairwise with mean $\pm \mathrm{SE}$ : $85 \pm 22(\mathrm{ng}), Z=-2.53, p=0.01$ and $497 \pm 167(\mathrm{ng} / \mathrm{mg})$, $Z=-2.53, p=0.01]$. Furthermore, there was no effect of egg mass on total egg (-)-adaline content $\left[X_{1}^{2}=1.33, p=0.25\right]$, but for those females that laid eggs in the absence of aphids 
TABLE 1 I Summary of the results of the effect of predation risk, aphid presence, the interactive effect of predation risk and aphid presence, and the effect of female age on the movement and egg laying behavior of female ladybirds during the experiment.

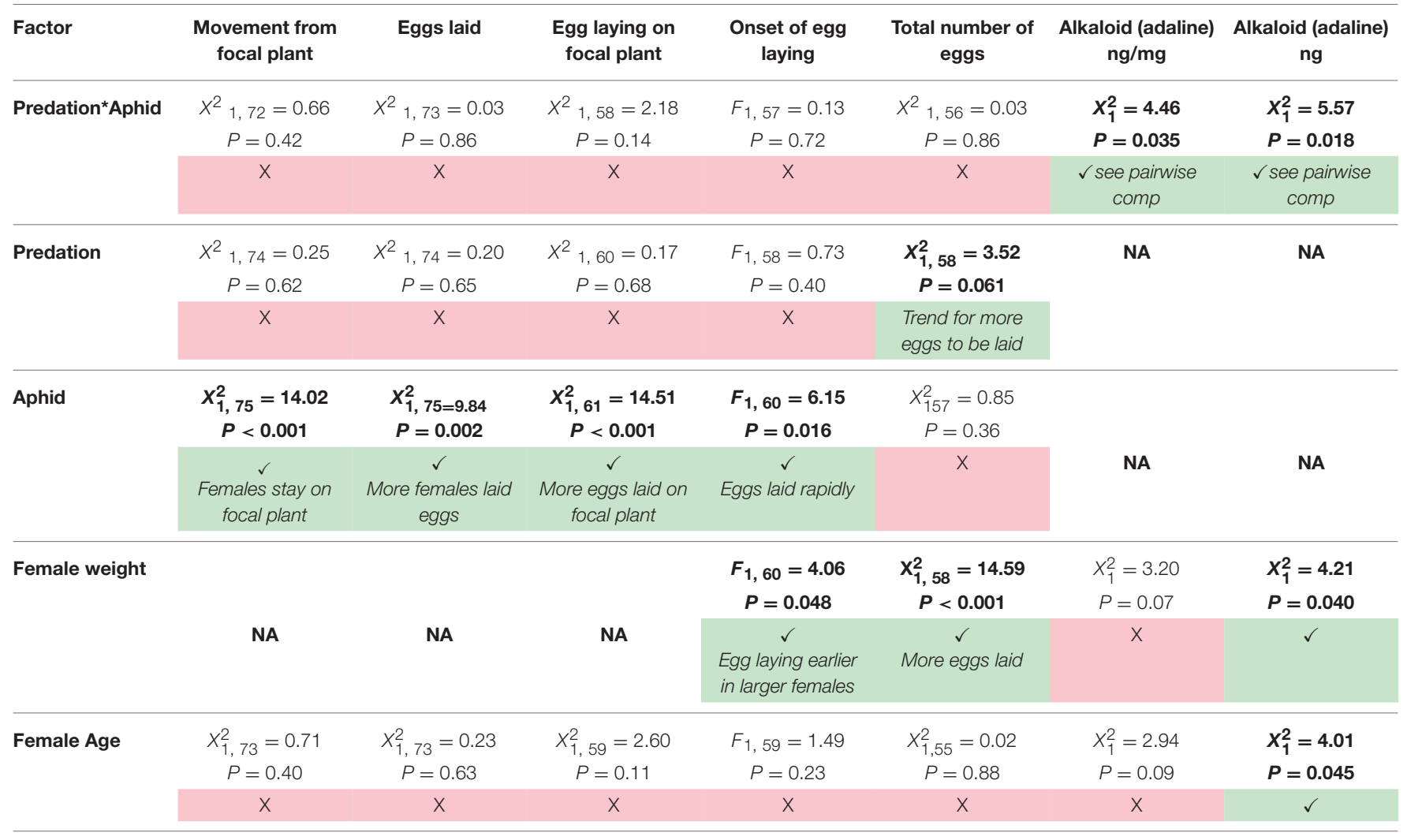

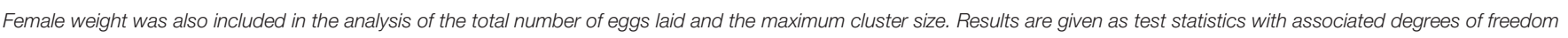
and p-value. Significant or near significant factors highlighted in green and bold.

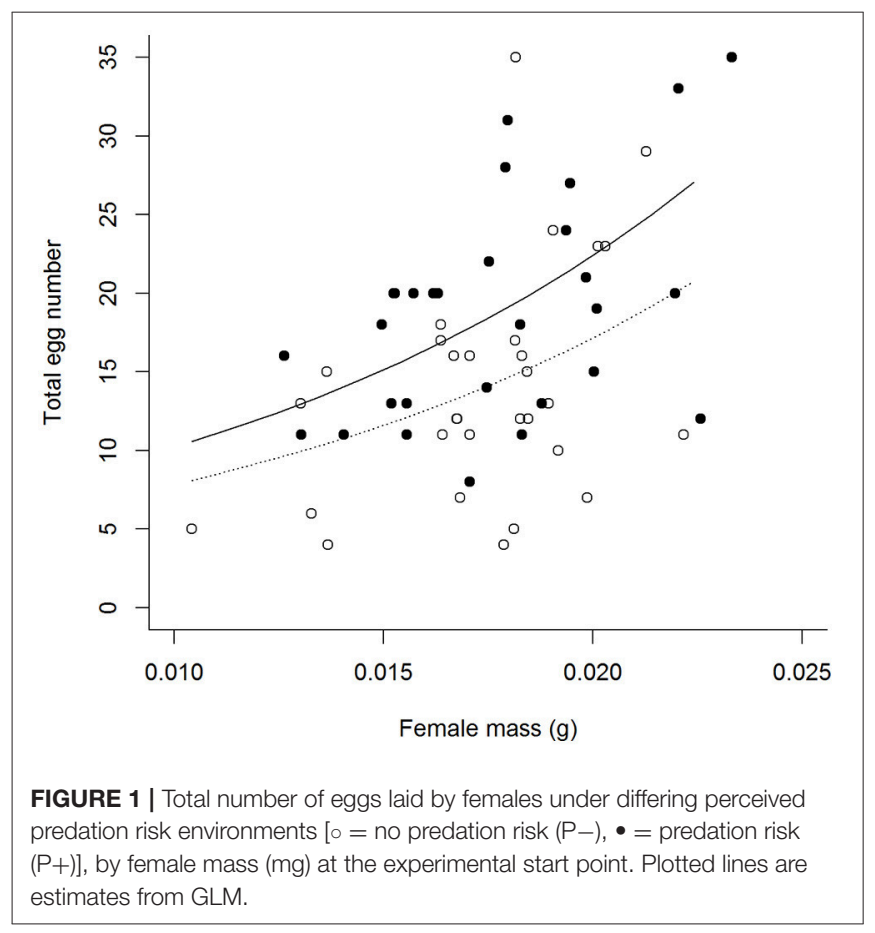

(i.e., $\mathrm{P}-/ \mathrm{A}-$ and $\mathrm{P}+/ \mathrm{A}-$ treatments) there was a significant negative correlation between egg (-)-adaline concentration and the total number of eggs laid [Figure $4 ; X_{1}^{2}=4.89$, $p=0.027$.

\section{Cannibalism Experiment}

One hundred and thirty-two out of the 161 larvae tested consumed an egg and, of the eggs eaten, significantly more contained high alkaloid levels $(\mathrm{H})(P=0.018$, probability of consuming $\mathrm{H}$ egg $=0.6, C I=0.52-0.69)$, but larval body condition did not affect egg choice $\left[X_{1}^{2}=0.23 p=0.63\right]$.

\section{DISCUSSION}

In egg laying species with no parental care, such as A. bipunctata, females employ two main strategies to maximize reproductive success in the face of variation in offspring predation risk and resource availability; finely tuned laying site choice (Refsnider and Janzen, 2010) and the alteration of egg phenotype (nongenetic maternal effects; Marshall and Uller, 2007). These nonmutually exclusive strategies are used by species in response to the complex gradients of variability in the quality and availability of sites on which to lay their eggs (Deas and Hunter, 2013, 2014). 


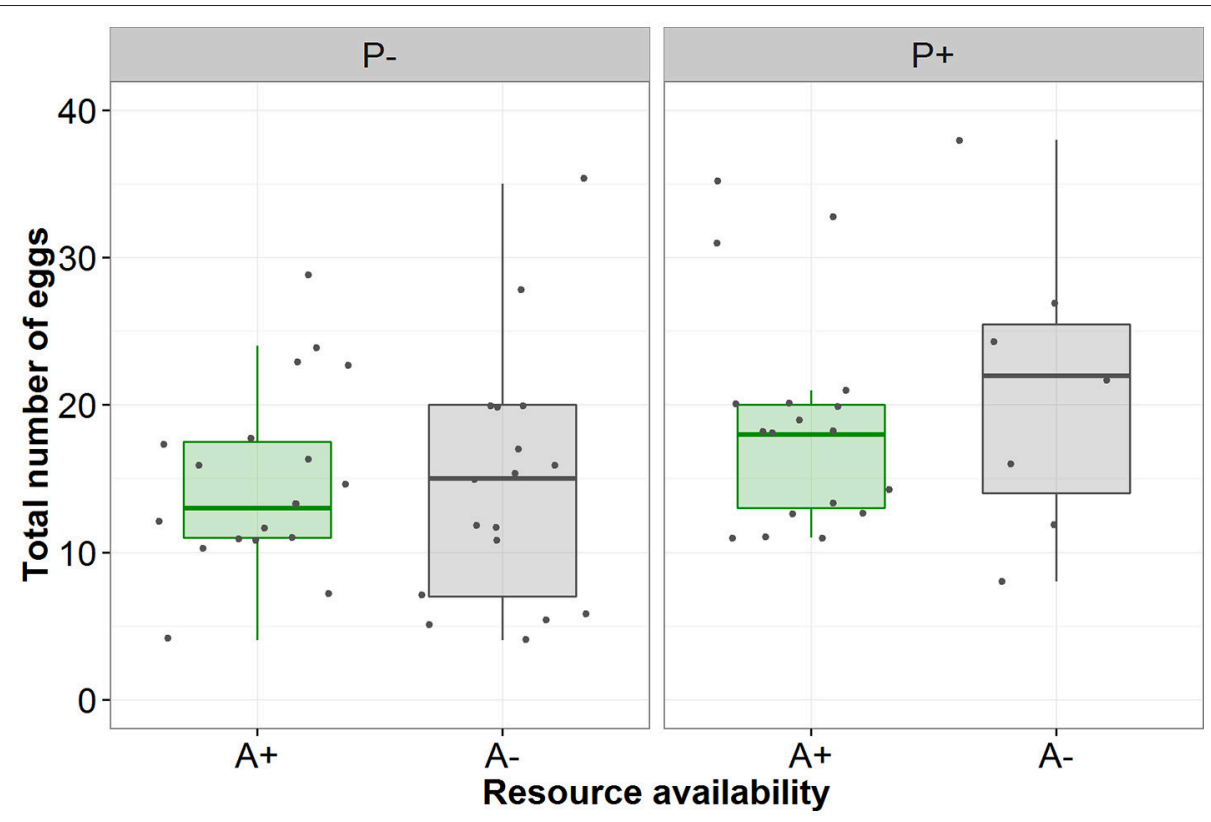

FIGURE 2 | The total number of eggs laid by females under conditions of no perceived predation risk ( $\mathrm{P}-$ ) or perceived predation risk $(\mathrm{P}+)$ and aphid presence (green/A+) or absence (gray/A-).

Females of multiple species alter laying site in response to cues of offspring predation risk (Refsnider and Janzen, 2010). This risk is rarely independent of other environmental factors that influence offspring survival, e.g., competition, which in turn can influence a female's reproductive response to offspring predation (e.g., Binckley and Resetarits, 2008). In female A. bipunctata ladybirds, however, predation risk and resource availability did not interact to affect the location or timing of female egg laying. Females were more likely to lay eggs, and lay eggs quickly, in the presence of aphids, irrespective of predator cues, emphasizing the importance of resource abundance in determining egg laying in ladybirds (Michaud and Jyoti, 2007).

In contrast, resource availability and predation risk interacted to affect egg (-)-adaline level (the alkaloid in A. bipunctata eggs). First and foremost the results of the A. bipunctata egg cannibalism experiment indicated that, as predicted, there is antagonism between the role of ladybird egg alkaloid level as a predator deterrent and cannibal attractant. Conspecific larval predators (cannibals) preferred eggs with high (-)-adaline levels over eggs with lower (-)-adaline levels. This result is consistent with the finding that, in addition to the general benefits of cannibalism (Ware et al., 2009), cannibalistic larvae that consume high alkaloid eggs have greater alkaloid levels themselves (Kajita et al., 2010), thus promoting survival (Marples et al., 1994). The role of egg alkaloid level and toxicity in determining consumption by heterospecific ladybird larvae is well-known (Katsanis et al., 2013), but this is the first demonstration that cannibals distinguish between, and preferentially consume, eggs with a high toxin level. The contribution of additional egg attributes, other than egg mass (see methods), to the preference shown by cannibalistic larvae, e.g., carotenoids (Winters et al.,
2014), cannot be ruled out. However, the highly chemically motivated nature of larvae (Cottrell, 2007) and the positive fitness impacts of consuming high alkaloid content eggs (Kajita et al., 2010), strongly support the contention that: (a) egg alkaloid levels play a role in the determination of egg consumption by cannibals, and (b) there is therefore a conflict between the optimal egg alkaloid level when resources are abundant and the optimal egg alkaloid level when egg predation risk is high.

Under such conditions we predicted that egg (-)-adaline levels would be greatest when perceived predation risk and the selective benefit of cannibalism are at their highest (i.e., $\mathrm{P}+/ \mathrm{A}-$ ) and smallest under the reverse conditions (i.e., $\mathrm{P}-/ \mathrm{A}+$ ). Previous work on individual phenotypic plasticity has demonstrated such graded responses to antagonistic selection pressures on optimal phenotype, e.g., in the freshwater snail Helisoma trivolvis where different predators select for differing shell morphology (shell thickness vs. width; Hoverman and Relyea, 2016). However, although there was an interactive effect of resource availability and cues of predation risk on egg (-)-adaline content, it was not in the direction predicted. Egg (-)-adaline level was greatest in the absence of aphids but, contrary to predictions, this was only the case when cues of predation risk were absent (as opposed to present) at the site where eggs were laid (i.e., $\mathrm{P}-/ \mathrm{A}-$ ).

That egg (-)-adaline level was highest when aphids were absent, i.e., when resources were low and the maternal fitness benefits of offspring cannibalism were high, further supports the idea that egg alkaloids play a role in sibling cannibalism. Although consistently beneficial to the cannibalistic offspring, it does not benefit maternal fitness, or the fitness of the siblings that are eaten, for offspring to cannibalize when resources are abundant enough for fecundity to be maximized 


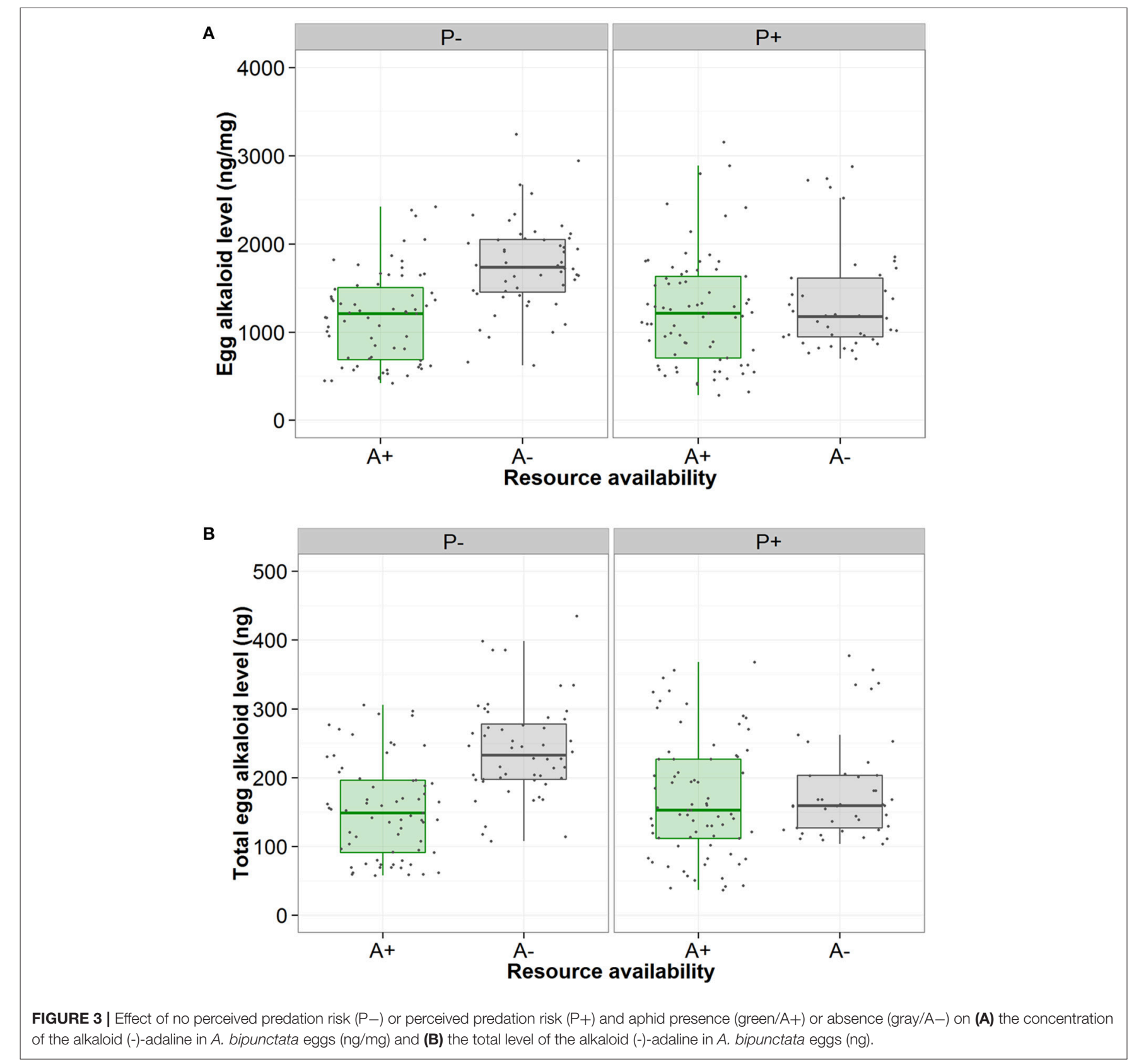

without it (Hamilton, 1964; Pfennig, 1997). This parent-offspring conflict has resulted in the evolution of mechanisms whereby mothers can manipulate levels of cannibalism in response to environmental conditions (Crespi, 1992). For example, mothers can increase the number of available offspring to be cannibalized by increasing hatching asynchrony (Michaud and Grant, 2004). Here, by increasing egg (-)-adaline content when aphids were absent, A. bipunctata mothers altered offspring phenotype in a way which would have potentially increased levels of sibling cannibalism under conditions where cannibalism was beneficial for maternal fitness. These results add to an emerging body of work examining other mechanisms by which levels of cannibalism are maternally fine-tuned (Wong et al., 2014), but are the first to indicate that an alteration in offspring "quality" (alkaloid level) can be used by females to promote levels of cannibalism, i.e., a "selfish" maternal effect (Marshall and Uller, 2007). It is also worth noting that hatching asynchrony occurs in other ladybird species (Perry and Roitberg, 2005). It has not previously been detected in A. bipunctata and is not directly investigated here, as egg toxin analysis is destructive, however, future studies could investigate whether it may work in concert with egg toxin level to influence sibling cannibalism level.

Considering the benefits of offspring cannibalism to maternal fitness under low resources, it is not immediately clear why 


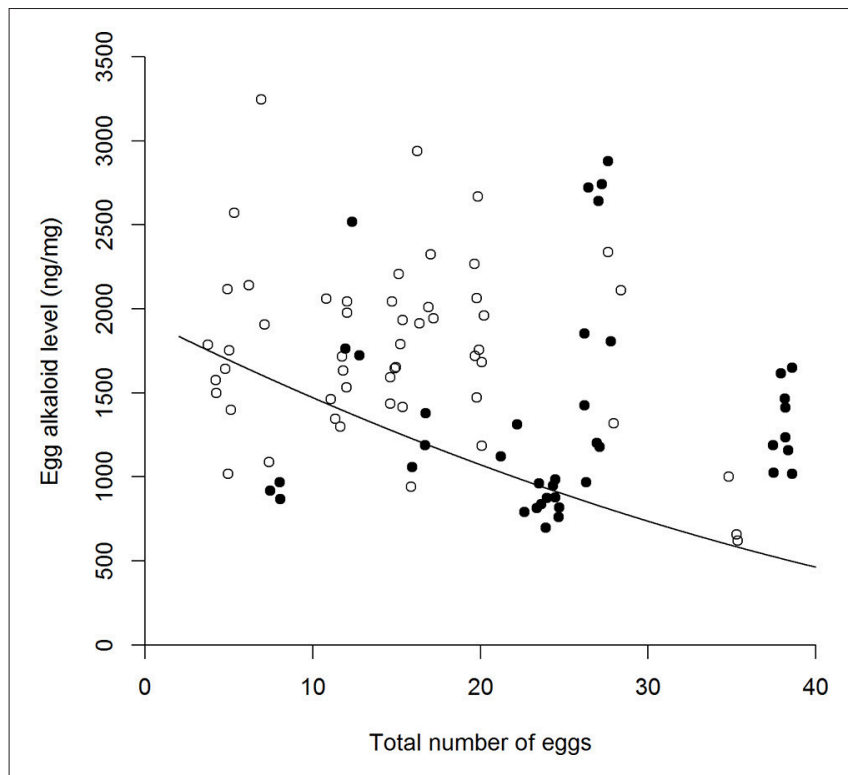

FIGURE 4 | Relationship between the total number of eggs laid by $A$. bipunctata females and the concentration of the alkaloid (-)-adaline in those eggs $(\mathrm{ng} / \mathrm{mg})$, in the absence of aphids $(A-)$ under different predation risk conditions $[0=$ no predation risk $(\mathrm{P}-), \bullet=$ predation risk $(\mathrm{P}+)]$. Plotted line represents GLM estimates.

increases in egg alkaloid level were observed in the absence of aphids only when predators were also absent. One explanation may be found in the trend for a greater number of eggs to be laid in the presence of predator cues, reflecting results from previous studies where A. bipunctata laid a larger number of eggs in response to the same stimulus (Paul et al., 2015). This suggests that females may have been constrained from any concomitant increase in investment in egg alkaloid level (Smith and Fretwell, 1974), resulting in the lack of increase in egg alkaloid level when aphids were absent but predator cues were present (i.e., $\mathrm{P}+/ \mathrm{A}-$ ). Classic life-history theory predicts a context dependent trade-off between offspring number and levels of per-offspring maternal investment (e.g., size; Parker and Begon, 1986; Bernardo, 1996b), that has repeatedly been empirically demonstrated. For example, the seed beetle Strator limbatus lays fewer but larger eggs when laying on tough seeds compared to pliable seeds; the larger eggs developing into larger larvae that are more likely to penetrate the thick seed coat (Fox et al., 1997). Here, though the trend for increasing egg number under high predation risk was marginal but non-significant, the significant negative correlation between the number of eggs laid and egg alkaloid level in the absence of aphids $(\mathrm{A}-)$, is indicative of a trade-off. This trade-off between egg number and egg alkaloid content suggests that predation risk may constrain the reproductive response of $A$. bipunctata to the trophic environment and further emphasizes the context dependent nature of maternal effects.

Alternatively, although it seems unlikely, we cannot rule out the possibility that a facultative reduction in female investment in environments of high offspring predation risk, may also have caused the observed difference in egg alkaloid level between the different predator treatments when aphids were absent. The total reproductive capacity of females is finite and therefore a reduction in offspring investment at risky or poor egg laying sites can enable them to increase their investment at more favorable sites, thus maximizing female fitness (Rosenheim, 1999). This does not necessarily involve the cessation of egg laying at risky sites, but a reduction, for example in offspring number (Guo et al., 2014), or in a costly aspect of offspring phenotype (Deas and Hunter, 2014). However, if $A$. bipunctata females were employing this strategy in the face of predation risk a reduction in egg alkaloid levels between predator treatments when aphids were present as well as absent, and in other markers of investment, such as egg size or number, may reasonably have been expected, but this was not seen.

In conclusion, there is an interactive effect of resource availability and invasive predator risk on maternal reproductive investment in A. bipunctata, but not in the direction predicted. The results from the cannibalism test and the increase in egg alkaloid levels in the absence of aphids and predators, indicates that egg alkaloid levels are used by female A. bipunctata to manipulate offspring cannibalism levels in response to low resource availability. Increased offspring predation risk also influences maternal response to low resource availability, with egg alkaloid levels being lower when simulated predation risk was high. The apparent trade-off between egg number and egg alkaloid level when resources are low, suggests that the lower level of egg alkaloids under is mediated by possible increases in egg number opposed to a facultative reduction in investment. Furthermore, the response of females to invasive predator tracks shown here is unlikely to be adequate to prevent detrimental effects of harlequin larval predation on A. bipunctata reproduction. Not least due to the higher tolerance of $H$. axyridis larvae to alkaloids (Sato and Dixon, 2004; Ware et al., 2009; Katsanis et al., 2013). Indeed, the decline in UK populations of $A$. bipunctata in regions where the invasive harlequin has become established supports this tentative conclusion (Roy et al., 2012). Overall these results emphasize the importance of studying the non-genetic transgenerational responses of species to anthropogenic change, such as those mediated by maternal effects, in the context of the other environmental factors key to determining offspring phenotype.

\section{ETHICS STATEMENT}

This study was carried out in accordance with the recommendations of the University of Exeter Ethics Committee and the Association for the Study of Animal Behavior. The protocol was approved by the the University of Exeter Ethics Committee.

\section{AUTHOR CONTRIBUTIONS}

SP, JDB, MS, and JP conceived and designed the experiments. SP, $\mathrm{JB}$, and $\mathrm{MB}$ were all involved in data collection. SP analyzed the data. SP, JDB, and MS wrote the manuscript with input from MB and JP. 


\section{FUNDING}

SP was funded by a Natural Environment Research Council PhD studentship (number: NER/K5009024/1). The funders had no role in study design, data collection and analysis, decision to publish, or preparation of the manuscript.

\section{REFERENCES}

Agarwala, B. K., and Dixon, A. F. G. (1992). Laboratory study of cannibalism and interspecific predation in ladybirds. Ecol. Entomol. 17, 303-309. doi: 10.1111/j.1365-2311.1992.tb01062.x

Banks, P. B., and Dickman, C. R. (2007). Alien predation and the effects of multiple levels of prey naïveté. Trends Ecol. Evol. 22, 229-230. doi: 10.1016/j.tree.2007.02.006

Bates, D., Maechler, M., Bolker, B., and Walker, S. (2015). Fitting linear mixedeffects models using lme4. J. Stat. Softw. 67, 1-48. doi: 10.18637/jss.v067.i01

Bernardo, J. (1996a). Maternal effects in animal ecology. Am. Zool. 36, 83-105. doi: $10.1093 / \mathrm{icb} / 36.2 .83$

Bernardo, J. (1996b). The particular maternal effect of propagule size, especially egg size: patterns, models, quality of evidence and interpretations. Am. Zool. 36, 216-236. doi: 10.1093/icb/36.2.216

Binckley, C. A., and Resetarits, W. J. Jr. (2008). Oviposition behavior partitions aquatic landscapes along predation and nutrient gradients. Behav. Ecol. 19, 552-557. doi: 10.1093/beheco/arm 164

Carter, M. C., and Dixon, A. F. G. (1982). Habitat quality and the foraging behaviour of coccinellid larvae. J. Anim. Ecol. 51, 865-878. doi: 10.2307/4011

Carthey, A. J. R., and Banks, P. B. (2014). Naïveté in novel ecological interactions: lessons from theory and experimental evidence. Biol. Rev. 89, 932-949. doi: $10.1111 /$ brv.12087

Cottrell, T. E. (2007). Predation by adult and larval lady beetles (Coleoptera: Coccinellidae) on initial contact with lady beetle eggs. Environ. Entomol. 36, 390-401. doi: 10.1093/ee/36.2.390

Cox, J. G., and Lima, S. L. (2006). Naivete and an aquatic-terrestrial dichotomy in the effects of introduced predators. Trends Ecol. Evol. 21, 674-680. doi: 10.1016/j.tree.2006.07.011

Crawley, M. J. (2014). The R book. Chichester: John Wiley \& Sons.

Crespi, B. J. (1992). "Cannibalism and trophic eggs in subsocial and eusocial insects," in Cannibalism: Ecology and Evolution among Diverse Taxa, eds M. A. Elgar and B. J. Crespi (Oxford: Oxford University Press), $176-213$.

Deas, J. B., and Hunter, M. S. (2013). Delay, avoidance and protection in oviposition behaviour in response to fine-scale variation in egg parasitism risk. Anim. Behav. 86, 933-940. doi: 10.1016/j.anbehav.2013.08.010

Deas, J. B., and Hunter, M. S. (2014). Egg and time limitation mediate an egg protection strategy. J. Evol. Biol. 27, 920-928. doi: 10.1111/jeb.12363

Donelson, J. M., Munday, P. L., McCormick, M. I., and Pitcher, C. R. (2012). Rapid transgenerational acclimation of a tropical reef fish to climate change. Nat. Clim. Change 2, 30-32. doi: 10.1038/nclimate1323

Doumbia, M., Hemptinne, J. L., and Dixon, A. F. G. (1998). Assessment of patch quality by ladybirds: role of larval tracks. Oecologia 113, 197-202. doi: $10.1007 / \mathrm{s} 004420050368$

Ferrari, M. C. O., Gonzalo, A., Messier, F., and Chivers, D. P. (2007). Generalization of learned predator recognition: an experimental test and framework for future studies. Proc. R. Soc. B Biol. Sci. 274, 1853-1859. doi: $10.1098 / \mathrm{rspb} .2007 .0297$

Fox, C. W., and Mousseau, T. A. (1996). Larval host plant affects fitness consequences of egg size variation in the seed beetle Stator limbatus. Oecologia 107, 541-548. doi: 10.1007/BF00333946

Fox, C. W., Thakar, M. S., and Mousseau, T. A. (1997). Egg size plasticity in a seed beetle: an adaptive maternal effect. Am. Naturalist 149, 149-163. doi: $10.1086 / 285983$

Guo, H., Meng, L., Wang, Y., Zheng, L., and Li, B. (2014). Oviposition behavior of the predatory midge Aphidoletes aphidimyza in response to aphid patch quality. J. Insect Behav. 27, 816-825. doi: 10.1007/s10905-014-9473-1

\section{ACKNOWLEDGMENTS}

Many thanks to J. Baverstock, S. Dewhirst, and J. Caulfield for their lab support. W. Hentley and R. Comont for assistance in sourcing harlequins and M. J. Silk and two reviewers for helpful comments on an earlier version of the manuscript.

Hamilton, W. D. (1964). Genetical evolution of social behaviour. J. Theor. Biol. 7, 17-52. doi: 10.1016/0022-5193(64)90039-6

Harrison, X. A. (2014). Using observation-level random effects to model overdispersion in count data in ecology and evolution. Peerj 2:e616. doi: $10.7717 /$ peerj. 616

Hill, R. K., and Renbaum, L. A. (1982). Assymetric syntheses of the ladybug alkaloid adaline and 1-methyl-9-azabicyclo [3.3. 1] nonan-3-one. Tetrahedron, 38, 1959-1963. doi: 10.1016/0040-4020(82)80045-8

Hothorn, T., Bretz, F., and Westfall, P. (2008). Simultaneous inference in general parametric models. Biom. J. 50, 346-363. doi: 10.1002/bimj.200810425

Hoverman, J. T., and Relyea, R. A. (2016). Prey responses to fine-scale variation in predation risk from combined predators. Oikos 125, 254-261. doi: 10.1111/oik.02435

Kajita, Y., Obrycki, J. J., Sloggett, J. J., and Haynes, K. F. (2010). Intraspecific alkaloid variation in ladybird eggs and its effects on con- and hetero-specific intraguild predators. Oecologia 163, 313-322. doi: 10.1007/s00442-009-1551-2

Katsanis, A., Babendreier, D., Nentwig, W., and Kenis, M. (2013) Intraguild predation between the invasive ladybird Harmonia axyridis and non-target European coccinellid species. Biocontrol 58, 73-83. doi: 10.1007/s10526-012-9470-2

Kovalenko, K. E., Dibble, E. D., Agostinho, A. A., and Pelicice, F. M. (2010). Recognition of non-native peacock bass, Cichla kelberi by native prey: testing the naiveté hypothesis. Biol. Invasions 12, 3071-3080. doi: 10.1007/s10530-010-9698-7

Lau, J. A., Peiffer, J., Reich, P. B., and Tiffin, P. (2008). Transgenerational effects of global environmental change: long-term $\mathrm{CO}_{2}$ and nitrogen treatments influence offspring growth response to elevated $\mathrm{CO}_{2}$. Oecologia 158, 141-150. doi: 10.1007/s00442-008-1127-6

Mack, R. N., Simberloff, D., Lonsdale, W. M., Evans, H., Clout, M., and Bazzaz, F. A. (2000). Biotic invasions: causes, epidemiology, global consequences, and control. Ecol. Appl. 10, 689-710. doi: 10.1890/10510761(2000)010[0689:BICEGC]2.0.CO;2

Magro, A., Tene, J. N., Bastin, N., Dixon, A. F. G., and Hemptinne, J.-L. (2007). Assessment of patch quality by ladybirds: relative response to conspecific and heterospecific larval tracks a consequence of habitat similarity? Chemoecology 17, 37-45. doi: 10.1007/s00049-006-0357-5

Marples, N. M., Vanveelen, W., and Brakefield, P. M. (1994). The relative importanace of color, taste, and smell in the protection of an aposematic insect Coccinella septempunctata. Anim. Behav. 48, 967-974. doi: 10.1006/anbe.1994.1322

Marshall, D. J., and Uller, T. (2007). When is a maternal effect adaptive? Oikos 116, 1957-1963. doi: 10.1111/j.2007.0030-1299.16203.x

Michaud, J. P., and Grant, A. K. (2004). Adaptive significance of sibling egg cannibalism in Coccinellidae: Comparative evidence from three species. Ann. Entomol. Soc. Am. 97, 710-719. doi: 10.1603/00138746(2004)097[0710:ASOSEC]2.0.CO;2

Michaud, J. P., and Jyoti, J. L. (2007). Repellency of conspecific and heterospecific larval residues to Hippodamia convergens (Coleoptera: Coccinellidae) ovipositing on sorghum plants. Eur. J. Entomol. 104, 399-405. doi: 10.14411/eje.2007.059

Miller, G. M., Watson, S.-A., Donelson, J. M., McCormick, M. I., and Munday, P. L. (2012). Parental environment mediates impacts of increased carbon dioxide on a coral reef fish. Nat. Clim. Change 2, 858-861. doi: 10.1038/nclimate1599

Mousseau, T. A., and Fox, C. W. (1998). The adaptive significance of maternal effects. Trends Ecol. Evol. 13, 403-407. doi: 10.1016/S0169-5347(98)01472-4

Nakagawa, S., and Schielzeth, H. (2010). Repeatability for Gaussian and nonGaussian data: a practical guide for biologists. Biol. Rev. Camb. Philos. Soc. 85, 935-956. doi: 10.1111/j.1469-185X.2010.00141.x 
Oliver, T. H., Timms, J. E. L., Taylor, A., and Leather, S. R. (2006). Oviposition responses to patch quality in the larch ladybird Aphidecta obliterata (Coleoptera: Coccinellidae): effects of aphid density, and con- and heterospecific tracks. Bull. Entomol. Res. 96, 25-34. doi: 10.1079/BER2005395

Otto, M., and Mackauer, M. (1998). The developmental strategy of an idiobiont ectoparasitoid, Dendrocerus carpenteri: influence of variations in host quality on offspring growth and fitness. Oecologia 117, 353-364. doi: $10.1007 / s 004420050668$

Paolucci, E. M., MacIsaac, H. J., and Ricciardi, A. (2013). Origin matters: alien consumers inflict greater damage on prey populations than do native consumers. Divers. Distributions 19, 988-995. doi: 10.1111/ddi.12073

Parker, G. A., and Begon, M. (1986). Optimal egg size and clutch size-effects of environment and maternal phenotype. Am. Naturalist 128, 573-592. doi: $10.1086 / 284589$

Paul, S. C., Pell, J. K., and Blount, J. D. (2015). Reproduction in risky environments: the role of invasive egg predators in ladybird laying strategies. PLOS ONE 10:e0139404. doi: 10.1371/journal.pone.0139404

Peig, J., and Green, A. J. (2009). New perspectives for estimating body condition from mass/length data: the scaled mass index as an alternative method. Oikos 118, 1883-1891. doi: 10.1111/j.1600-0706.2009.17643.x

Peig, J., and Green, A. J. (2010). The paradigm of body condition: a critical reappraisal of current methods based on mass and length. Funct. Ecol. 24, 1323-1332. doi: 10.1111/j.1365-2435.2010.01751.x

Pell, J. K., Baverstock, J., Roy, H. E., Ware, R. L., and Majerus, M. E. (2008). Intraguild predation involving Harmonia axyridis: a review of current knowledge and future perspectives. BioControl 53, 147-168. doi: 10.1007/s10526-007-9125-x

Perry, J. C., and Roitberg, B. D. (2005). Ladybird mothers mitigate offspring starvation risk by laying trophic eggs. Behav. Ecol. Sociobiol. 58, 578-586. doi: 10.1007/s00265-005-0947-1

Pfennig, D. W. (1997). Kinship and cannibalism. Bioscience 47, 667-675. doi: $10.2307 / 1313207$

R Core Team (2014). R: A Language and Environment for Statistical Computing. Vienna: R Foundation for Statistical Computing. Available online at: http:// www.r-project.org/

Refsnider, J. M., and Janzen, F. J. (2010). Putting eggs in one basket: ecological and evolutionary hypotheses for variation in oviposition-site choice. Ann. Rev. Ecol. Evol. Syst. 41, 39-57. doi: 10.1146/annurev-ecolsys-102209-144712

Rehage, J. S., Dunlop, K. L., and Loftus, W. F. (2009). Antipredator responses by native mosquito fish to non-native cichlids: an examination of the role of prey naïveté. Ethology 115, 1046-1056. doi: 10.1111/j.1439-0310.2009.01694.x

Relyea, R. A. (2004). Fine-tuned phenotypes: tadpole plasticity under 16 combinations of predators and competitors. Ecology 85, 172-179. doi: 10.1890/03-0169

Rosenheim, J. A. (1999). The relative contributions of time and eggs to the cost of reproduction. Evolution 53, 376-385. doi: 10.1111/j.1558-5646.1999.tb03773.x

Roy, H. E., Adriaens, T., Isaac, N. J. B., Kenis, M., Onkelinx, T., San Martin, G., et al. (2012). Invasive alien predator causes rapid declines of native European ladybirds. Divers. Distributions 18, 717-725. doi: 10.1111/j.1472-4642.2012.00883.x

Ruzicka, Z. (2002). Persistence of deterrent larval tracks in Coccinella septempunctata, Cycloneda limbifer and Semiadalia undecimnotata (Coleoptera: Coccinellidae). Eur. J. Entomol. 99, 471-475. doi: $10.14411 /$ eje.2002.061
Sato, S., and Dixon, A. F. G. (2004). Effect of intraguild predation on the survival and development of three species of aphidophagous ladybirds: consequences for invasive species. Agric. Forest Entomol. 6, 21-24. doi: 10.1111/j.1461-9555.2004.00197.x

Smith, C. A., and Gardiner, M. M. (2013). Oviposition habitat influences egg predation of native and exotic coccinellids by generalist predators. Biol. Control 67, 235-245. doi: 10.1016/j.biocontrol.2013.07.019

Smith, C. C., and Fretwell, S. D. (1974). Optimal balance between size and number of offspring. Am. Naturalist 108, 499-506. doi: 10.1086/282929

Steffen, W., Crutzen, P. J., and McNeill, J. R. (2007). The Anthropocene: are humans now overwhelming the great forces of nature. AMBIO 36, 614-621.

Storm, J. J., and Lima, S. L. (2010). Mothers forewarn offspring about predators: a transgenerational maternal effect on behavior. Am. Naturalist 175, 382-390. doi: $10.1086 / 650443$

Tollrian, R., Duggen, S., Weiss, L. C., Laforsch, C., and Kopp, M. (2015). Density-dependent adjustment of inducible defenses. Sci. Rep. 5:12736. doi: $10.1038 /$ srep 12736

Touchon, J. C., and Worley, J. L. (2015). Oviposition site choice under conflicting risks demonstrates that aquatic predators drive terrestrial egglaying. Proc. R. Soc. B Biol. Sci. 282, 2015036. doi: 10.1098/rspb.20 15.0376

Visser, M. E. (2008). Keeping up with a warming world: assessing the rate of adaptation to climate change. Proc. R. Soc. Lond. B Biol. Sci. 275, 649-659. doi: $10.1098 /$ rspb.2007.0997

Walsh, M. R., Cooley, F., Biles, K., and Munch, S. B. (2015). Predatorinduced phenotypic plasticity within- and across-generations: a challenge for theory? Proc. R. Soc. B Biol. Sci. 282, 20142205. doi: 10.1098/rspb.2014. 2205

Ware, R., Yguel, B., and Majerus, M. (2009). Effects of competition, cannibalism and intra-guild predation on larval development of the European coccinellid Adalia bipunctata and the invasive species Harmonia axyridis. Ecol. Entomol. 34, 12-19. doi: 10.1111/j.1365-2311.2008.01036.x

Winters, A. E., Stevens, M., Mitchell, C., Blomberg, S. P., and Blount, J. D. (2014). Maternal effects and warning signal honesty in eggs and offspring of an aposematic ladybird beetle. Funct. Ecol. 28, 1187-1196. doi: $10.1111 / 1365-2435.12266$

Wolf, J. B., and Wade, M. J. (2009). What are maternal effects (and what are they not)? Philos. Trans. R. Soc. B Biol. Sci. 364, 1107-1115. doi: 10.1098/rstb.2008.0238

Wong, J. W. Y., Lucas, C., and Koelliker, M. (2014). Cues of maternal condition Influence offspring selfishness. PLoS ONE 9:e87214. doi: 10.1371/journal.pone.0087214

Conflict of Interest Statement: The authors declare that the research was conducted in the absence of any commercial or financial relationships that could be construed as a potential conflict of interest.

Copyright (c) 2018 Paul, Stevens, Burton, Pell, Birkett and Blount. This is an openaccess article distributed under the terms of the Creative Commons Attribution License (CC BY). The use, distribution or reproduction in other forums is permitted, provided the original author(s) and the copyright owner are credited and that the original publication in this journal is cited, in accordance with accepted academic practice. No use, distribution or reproduction is permitted which does not comply with these terms. 